\title{
THE EFFECT OF HEAT STRESS ON THE STANDARDS OF ACID BALANCE-THE BASEMENT FLAYING HENS
}

RANA A.ASEM

Department of Physiology, Biochemistry \& Pharmacology, College of Veterinary Medicine, University of Mosul, IRAQ Email: ranaamer2003@yahoo.com

\section{ABSTRACT}

Received at: 24/7/2014

Accepted: 16/11/2014
This study was designed to evaluate the effects of heat stress on the stability criteria of acid - base and electrolyte balance of laying hens, as well as assessing the impact of the regionalization on the mentioned criteria. Thirty six white leghorn chickens were used chickens fed balanced diets for production stage, bird were divided into three equal groups. The first group was control while the second group was adapted to $38^{\circ} \mathrm{C}$ for a period of ten days before the start of the experiment. The third group (non- adopted) was exposed to the temperature of $38^{\circ} \mathrm{C}$ (two hours per day) for a period of thirty days. The results of the statistical analysis $(\mathrm{p} \leq 0.05)$ to the adaptation have had a significant role in reducing the concentration of adrenocortical hormonal of the group and improve the level of serum hormone glutathaon the heat stress regardless of the adaptation had effect in raising the concentration of potassium ion and reducing calcium ion at the end of the experiment without affecting the level of sodium ion. Heat treatment also reduced the molecular pressure of carbon dioxide in both heat treated groups while the concentration of bicarbonates was elevated in non adapted group at the end of the As well as, the heat treatment in both groups led to lawering the volume of packed cells in both groups. The study concludes that heat stress has negative impact on the standards of the acid - base balance in laying hens and the effect of acclimatization period was limited in alleviating heat stress in chickens.

Key words: Heat stress, Acid-base balance, Flaying hens.

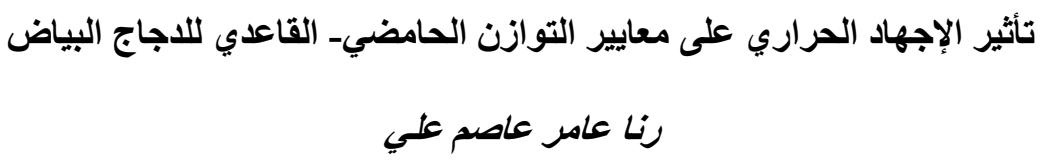

Email: ranaamer2003@yahoo.com

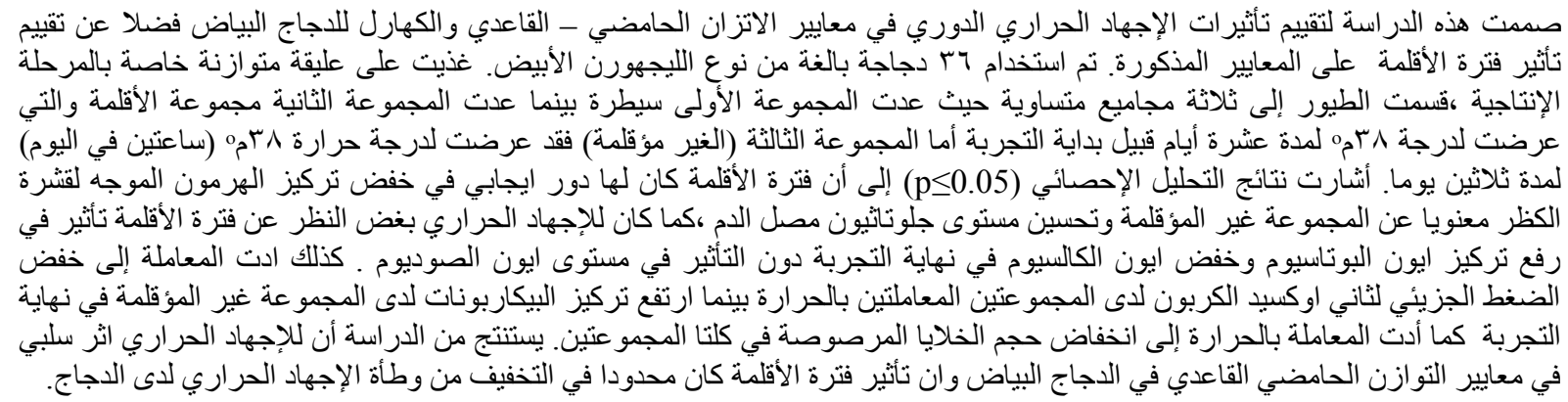

\section{INTRODUCTION}

المقدمــة

يعد الإجهاد الحراري heat stress في الدواجن كاستجابة لزيادة درجة حر ارة الجسم نتيجة لارتفاع درجة حرارة المحيط عن طريق الإيق

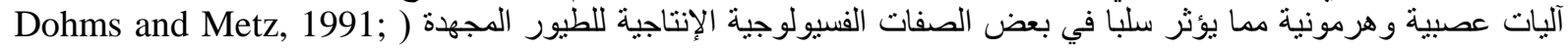


ينعكس الإجهاد الحر اري بصورة سلبية على وزن الجسم (Ramanth et al., 2007) واستهلاك العلف (Abd_Elsamee, 2005)

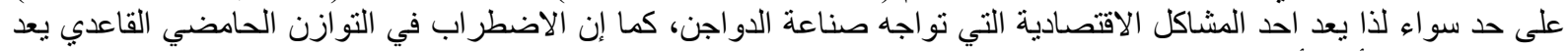

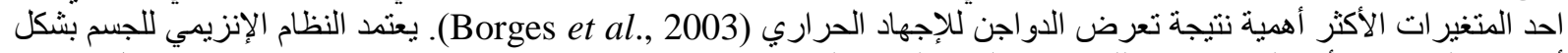

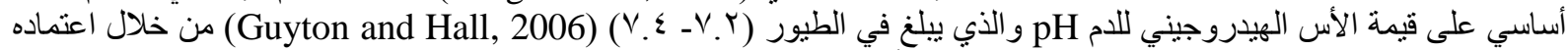

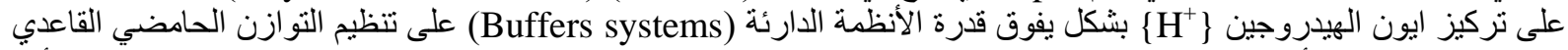

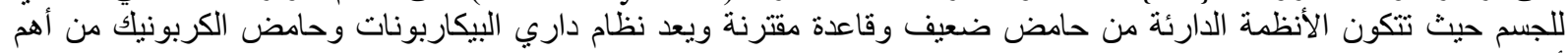
أنظمة الجسم الدارئة، هذا فضلا عن دور نظام دارى حامض الفئل الفسوريك و الفوسفات (Guyton and Hall, 2006).

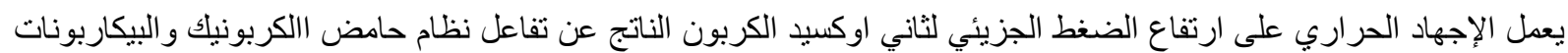

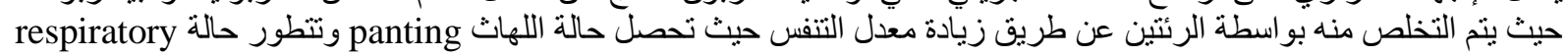
alkalosis

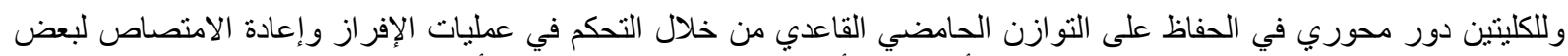

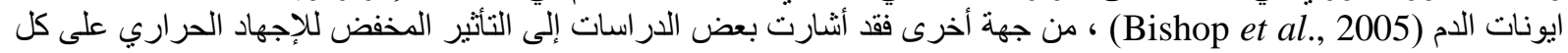

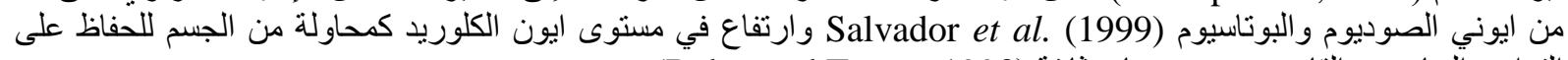

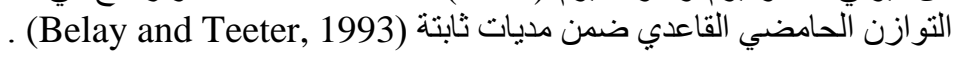

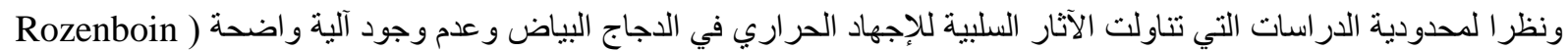

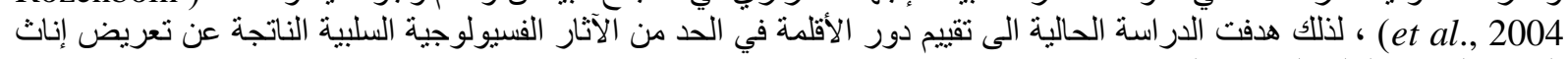
الاجاج البياض البالغة للإجهاد الحراري.

\section{MATERIALS and METHODS مواد وطرق البحث}

أجريت الدراسة في بيت الحيو انات المختبرية ، كلية الطب البيطري،جامعة الموصل واستمرت الفترة من 14/6/2010 وحتى 1 /8 / 2010

الحيوانات المستخدمة:

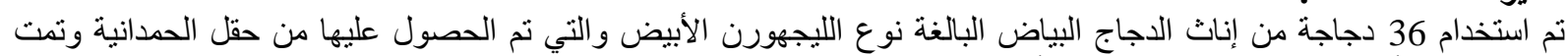

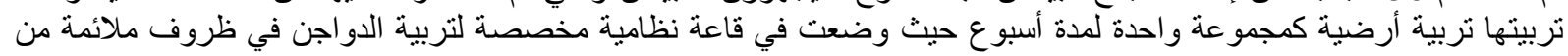

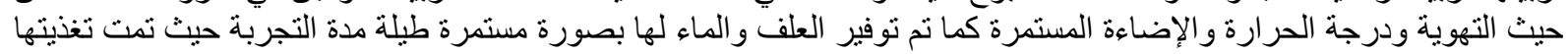

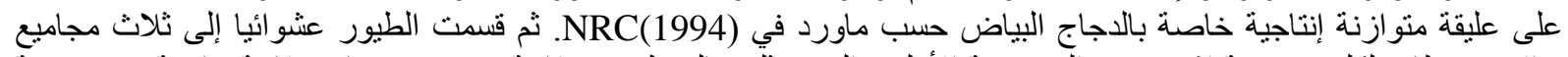

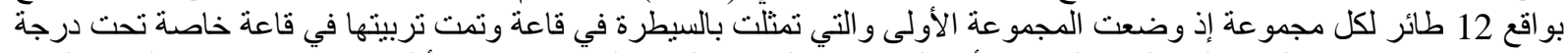

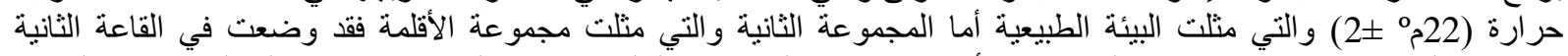

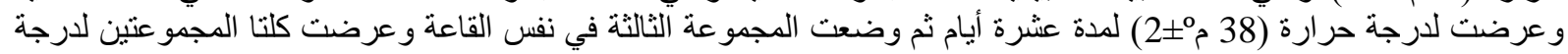

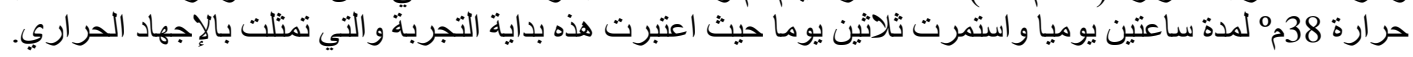

تصميم الدراسة: شملت الدراسة ثلاثة مجاميع وكما يلي:

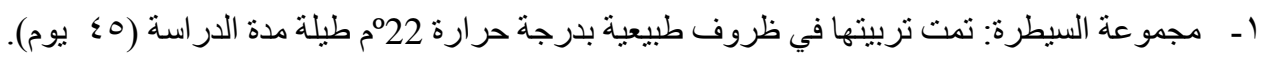

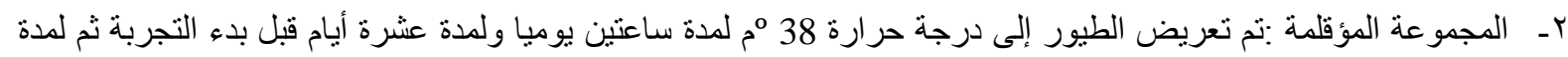

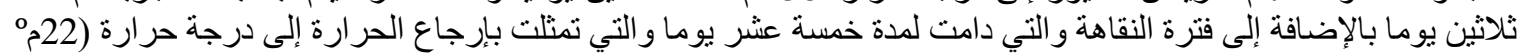

ץ- المجمو عة الغير المؤقلمة: هي المجموعة التي تم تعريضها إلى درجة حرارة 38 م بشكل مفاجئ ولمدة ثلاثين يوما بالإضافة

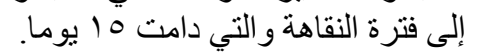

جمع العينات:

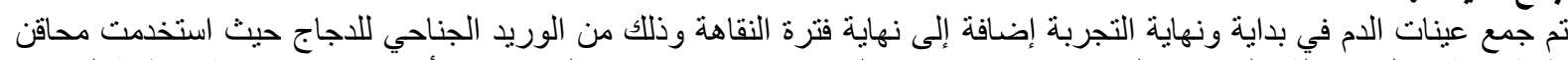

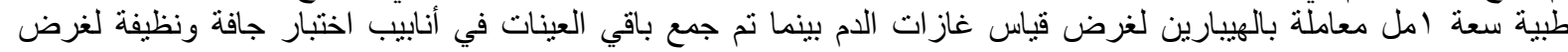

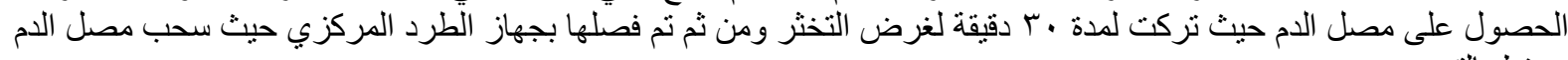




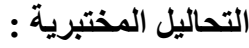

تم قياس غازات الدم ومعايير التوازن الحامضي القاعدي فضلا عن الكهارل بالسرعة المكنة باستخدام جهاز قياس غازات الدم Blood gas analyzer نوع (OPTICCA,USA). طريق تقنية التالق المناعيRadio immunoassay في مختبر الباب الثرفي في بغداد باد باستخدام عدة التحليل الخاصة

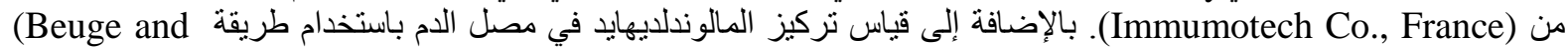
(Bust, 1978, Wysocka et al., 1995) وقياس تركيز الجلوناثيون باستخدام طريقة (Burits and Ashood, 1999).

تم تحليل البيانات باستخدام اختبار التداخل الثنائي Two Way Analysis of Variance واستخدام اختبار دنكن multiple test .(Steel and Torrie, 1980) $(\mathrm{P} \leq 0.05)$

\section{RESULTS النتائج}

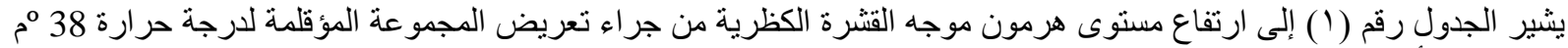

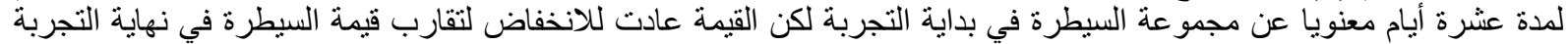
وفترة النقاهة بينما أظهرت المجموعة الغير مؤقلمة ارتفاعا معنويا في نهاية التجربة و الذي استمر خلال فترة النقاهة.

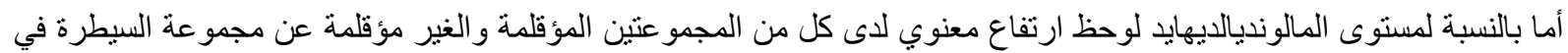

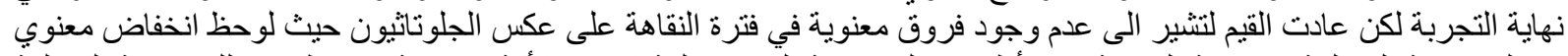

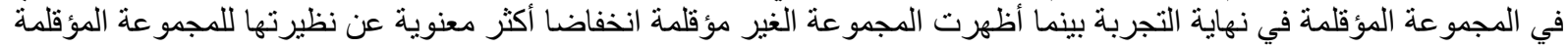

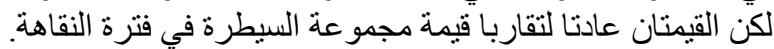
جلول رقم (1): تأثير الإجهاد الحراري والأقلمة في مستوى الهرمون الموجه لقترة الكظر و المالوندلديهايد و الكلوتاثايون في الدجاج البياض.

\begin{tabular}{|c|c|c|c|c|c|c|c|c|c|}
\hline \multicolumn{3}{|c|}{ 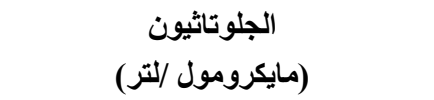 } & \multicolumn{3}{|c|}{ 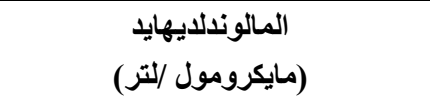 } & \multicolumn{4}{|c|}{ 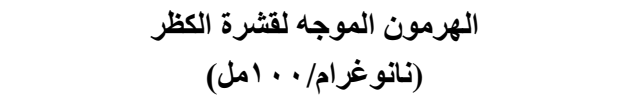 } \\
\hline النقاهة & التجرية & التجربة & النقاهة & التجربية & التجربية & النقاهة & التجربية & التجربة & المجموعة \\
\hline $\begin{array}{c}0.105 \\
\pm 0.012 \\
\text { A }\end{array}$ & $\begin{array}{c}0.07 \\
\pm 0.008 \\
\mathrm{~A}\end{array}$ & $\begin{array}{c}0.088 \\
\pm 0.0006 \\
\text { A }\end{array}$ & $\begin{array}{c}0.26 \\
\pm 0.05 \\
\text { B }\end{array}$ & $\begin{array}{c}0.2561 \\
\pm 0.014 \\
\text { B }\end{array}$ & $\begin{array}{c}0.1991 \\
\pm 0.0086 \\
\text { B }\end{array}$ & $\begin{array}{c}1.74 \\
\pm 0.90 \\
\text { B }\end{array}$ & $\begin{array}{c}1.75 \\
\pm 0.012 \\
\text { B }\end{array}$ & $\begin{array}{c}1.64 \\
\pm 0.09 \\
\text { B }\end{array}$ & المجيطوة عة \\
\hline $\begin{array}{c}0.08 \\
\pm 0.03 \\
\text { A }\end{array}$ & $\begin{array}{c}0.05 \\
\pm 0.03 \\
\text { B }\end{array}$ & $\begin{array}{c}0.0996 \\
\pm 0.011 \\
\text { A }\end{array}$ & $\begin{array}{c}0.1792 \\
\pm 0.004 \\
\text { B }\end{array}$ & $\begin{array}{c}0.2994 \\
\pm 0.011 \\
\text { A }\end{array}$ & $\begin{array}{c}0.1883 \\
\pm 0.036 \\
\text { B }\end{array}$ & $\begin{array}{c}1.61 \\
\pm 0.17 \\
\text { B }\end{array}$ & $\begin{array}{c}1.73 \\
\pm 0.06 \\
\text { B }\end{array}$ & $\begin{array}{c}0.361 \\
\pm 0.86 \\
\text { A }\end{array}$ & مجمو عة الاقلمة \\
\hline $\begin{array}{c}0.08 \\
\pm 0.019 \\
\text { A }\end{array}$ & $\begin{array}{c}0.03 \\
\pm 0.014 \\
\mathrm{C}\end{array}$ & $\begin{array}{c}0.084 \\
\pm 0.01 \\
\mathrm{~A}\end{array}$ & $\begin{array}{c}0.2164 \\
\pm 0.02 \\
\text { B }\end{array}$ & $\begin{array}{c}0.3194 \\
\pm 0.049 \\
\text { A }\end{array}$ & $\begin{array}{c}0.1834 \\
\pm \\
0.0255 \\
\text { В }\end{array}$ & $\begin{array}{c}3.28 \\
\pm 0.74 \\
\text { A }\end{array}$ & $\begin{array}{c}6.26 \\
\pm 0.73 \\
\mathrm{~A}\end{array}$ & $\begin{array}{c}1.67 \\
\pm 0.09 \\
\text { B }\end{array}$ & مؤقلمة المجمة الغير \\
\hline
\end{tabular}

الحروف المختلفة تغني فروقا معنوية عند مستوى احتمالية (10 • ..).

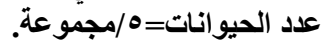
القيم معبر عنها المعدل 土ـ الخطأ القياسي

لم يتبين في الجدول رقم (r) أي اختلاف معنوي في ما يخص تركيز ايون الصوديوم لكن ايون البوتاسيوم اظهر ارتفاع معنوي في في إني

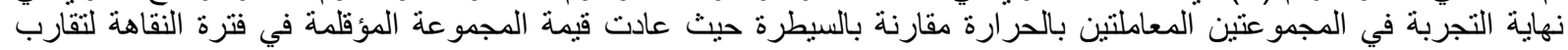

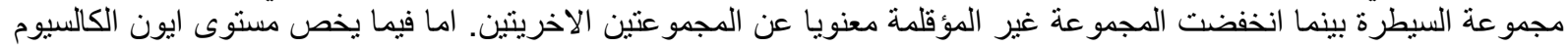

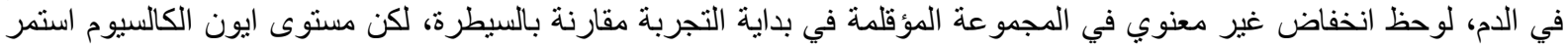

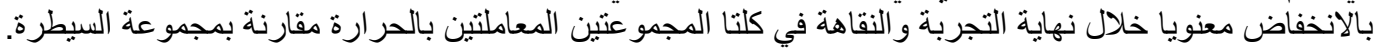


Assiut Vet. Med. J. Vol. 60 No. 143 October 2014

جدول رقم (ץ) : نأثير الإجهاد الحراري و الأقلمة في مستوى كهارل الدم.

\begin{tabular}{|c|c|c|c|c|c|c|c|c|c|}
\hline \multicolumn{3}{|c|}{ 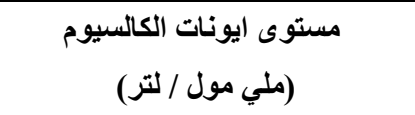 } & \multicolumn{3}{|c|}{ 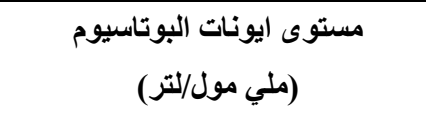 } & \multicolumn{4}{|c|}{ 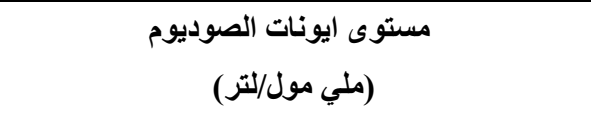 } \\
\hline فنترة & التجرية & التجربة & فنقاهة & التجرية & التجربية & فتلترة & التجرية & التجربة & المجموعة \\
\hline 1.13 & 1.15 & 1.21 & 4.18 & 4.52 & 4.68 & 146 & 136 & 145.8 & مجمو عة \\
\hline \pm 0.04 & \pm 0.11 & \pm 0.04 & \pm 0.26 & \pm 0.29 & \pm 0.27 & \pm 1.76 & \pm 6.90 & \pm 1.62 & \\
\hline A & A & A & B & B & B & A & A & A & \\
\hline 1.20 & 1.47 & 1.05 & 4.06 & 4.98 & 4.40 & 138 & 142 & 144.8 & مجمو عة \\
\hline \pm 0.05 & \pm 0.10 & \pm 0.12 & \pm 0.26 & \pm 0.35 & \pm 0.52 & \pm 2.96 & \pm 1.94 & \pm 2.08 & الاقلمة \\
\hline B & $\mathrm{B}$ & $\mathrm{AB}$ & B & A & B & $\mathrm{A}$ & A & A & \\
\hline 1.28 & 1.04 & 1.09 & 3.32 & 4.90 & 4.64 & 146 & 132.8 & 140.6 & المجمو عة \\
\hline \pm 0.04 & \pm 0.06 & \pm 0.07 & \pm 0.03 & \pm 0.14 & \pm 0.25 & \pm 2.50 & \pm 5.36 & \pm 1.56 & مئقالغيرة \\
\hline B & B & $\mathrm{AB}$ & $\mathrm{C}$ & A & B & $\mathrm{A}$ & A & A & \\
\hline
\end{tabular}

الحروف المختلفة تغني فروقا معنوية عند مستوى احتمالية (10 •. . ). عدد الحيوانات=|•/مجموعة. القيم معبر عنها المعدل \pm الخطأ القياسي

أما الجدول رقم (ب) فقد أثثير فيه إلى أن حجم الخلايا المرصوصة في المجمو عتين المعاملتين بالحر ارة قد اظهرتا انخفاضا معنويا

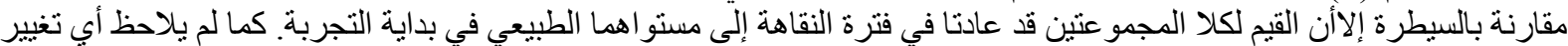
معنوي في قيم تركيز الهيموجلوبين طيلة فترة التجربة. جدول رقم (ّ): تأثئير الإجهاد الحراري والأقلمة في تركيز الهيموجلوبين والنسبة المئوية لحجم الخلايا المرصوصة في دم الدجاج

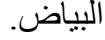

\begin{tabular}{|c|c|c|c|c|c|c|}
\hline \multicolumn{3}{|c|}{ الهيموجلوبين(غم/ · 1 مل دم) } & \multicolumn{4}{|c|}{ حجم الخلايا المرصوصة(\%) } \\
\hline فترة النقاهة & نهاية التجربة & بداية التجربة & فترة النقاهة & نهاية التجربة & بداية التجربة & المجموعة \\
\hline 8.12 & 8.32 & 8.07 & 36 & 35 & 37 & مجمو عة \\
\hline \pm 0.90 & \pm 0.012 & \pm 0.45 & \pm 0.94 & \pm 1.41 & \pm 1.30 & السيطرة \\
\hline A & A & A & A & A & A & \\
\hline 8.61 & 7.73 & 8.36 & 34 & 25.4 & 36 & مجمو عة الاقلمة \\
\hline \pm 0.17 & \pm 0.06 & \pm 0.86 & \pm 1.11 & \pm 0.74 & \pm 2.11 & \\
\hline A & A & A & A & $\mathrm{Bc}$ & A & \\
\hline 7.28 & 7.26 & 8.67 & 35 & 28.6 & 37 & المجمو عة الغير \\
\hline \pm 0.74 & \pm 0.73 & \pm 0.09 & \pm 0.84 & \pm 1.32 & \pm 0.86 & مؤقلمة \\
\hline A & A & A & A & $\mathrm{Bc}$ & A & \\
\hline
\end{tabular}

الحروف المختلفة تعني فروقا معنوية عند مستوى احتمالية (ا>>ه .. ·).

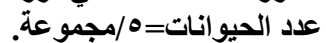
القيم معبر عنها المعدل \pm الخطأ القياسي

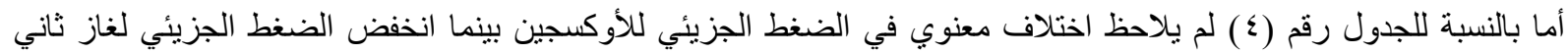

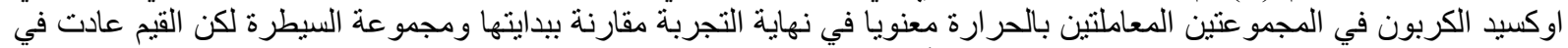

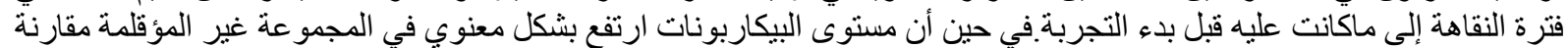

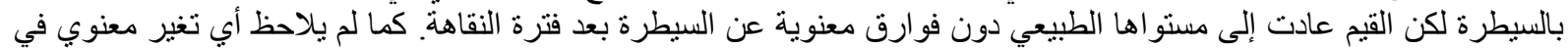
قيمة باها الدم. 
Assiut Vet. Med. J. Vol. 60 No. 143 October 2014

جدول رقم (๕) ): تأثير الإجهاد الحراري والأقلمة في معايير الاتزان الحامضي -القاعدي.

\begin{tabular}{|c|c|c|c|c|c|c|c|c|c|c|c|c|}
\hline \multicolumn{3}{|c|}{ pH } & \multicolumn{3}{|c|}{ Hco3(ملي مول/لتر) } & \multicolumn{3}{|c|}{ Pco2 (ملم زئبقي) } & \multicolumn{4}{|c|}{ Po2(ملم زئبقي) Po2 } \\
\hline النقاهة & التجرية & التجربة & النقاهة & التجرية & التجربة & فتقاهة & التجرية & التجربة & فتنرة & التهرية & التجربة & المجموعة \\
\hline 7.38 & 7.39 & 7.45 & 20.88 & 20.92 & 17.28 & 36.4 & 31.6 & 34 & 70 & 67.8 & 69.8 & مجموعة \\
\hline $0.02 \pm$ & $0.01 \pm$ & \pm 0.02 & $0.80 \pm$ & $1.20 \pm$ & \pm 1.13 & \pm 1.60 & $1.96 \pm$ & $1.81 \pm$ & $0.94 \pm$ & $2.63 \pm$ & \pm 0.78 & السيطرة \\
\hline A & A & A & $\mathrm{BC}$ & $\mathrm{BC}$ & $\mathrm{C}$ & A & A & A & A & A & A & \\
\hline 7.44 & 7.26 & 7.43 & 18 & 22.70 & 17.24 & 32 & 22.8 & 30.2 & 67.2 & 59.6 & 67 & مجموعة \\
\hline $0.007 \pm$ & $0.01 \pm$ & $0.01 \pm$ & $0.46 \pm$ & $1.04 \pm$ & $2.42 \pm$ & $0.44 \pm$ & $1.15 \pm$ & $2.50 \pm$ & $3.89 \pm$ & $2.83 \pm$ & $4.61 \pm$ & الاقلمة \\
\hline A & A & A & $\mathrm{C}$ & $\mathrm{B}$ & $\mathrm{C}$ & A & B & $\mathrm{A}$ & A & A & A & \\
\hline 7.51 & 7.25 & 7.56 & 17.48 & 29.54 & 17.08 & 34 & 19.8 & 33 & 73 & 54 & 73.2 & المجمو عة \\
\hline \pm 0.03 & $0.01 \pm$ & $0.04 \pm$ & $0.73 \pm$ & $0.07 \pm$ & \pm 0.98 & $2.54 \pm$ & $1.59 \pm$ & $2.09 \pm$ & $2.34 \pm$ & $2.70 \pm$ & $12.29 \pm$ & الغير مؤقلمة \\
\hline A & $\mathrm{A}$ & A & $\mathrm{C}$ & A & $\mathrm{C}$ & $\mathrm{A}$ & B & $\mathrm{A}$ & $\mathrm{A}$ & $\mathrm{A}$ & A & \\
\hline
\end{tabular}

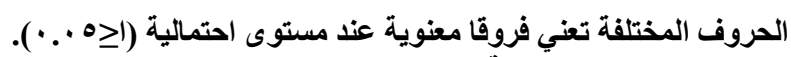

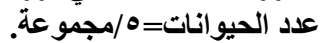

القيم معبر عنها المعدل \pm الخطأ القياسي

\section{DISCUSSION}

المناقثة

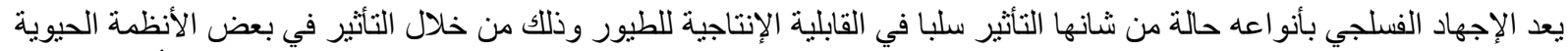

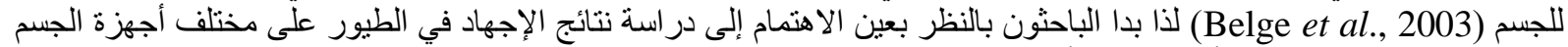

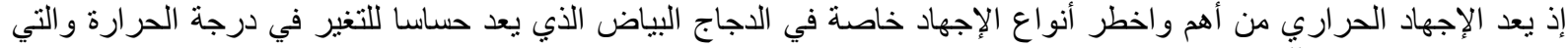

تؤثر في مدى إنتاجيته للبيض (Puvaldopirod \& Thaxon, 2000).

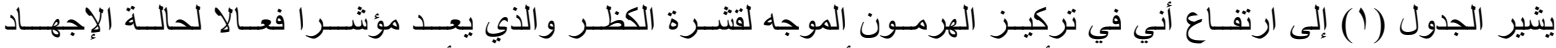

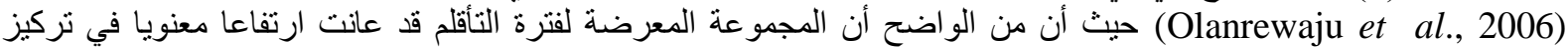

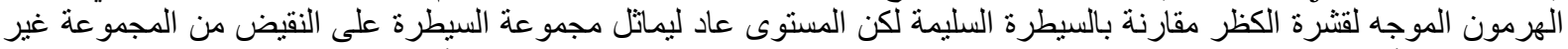

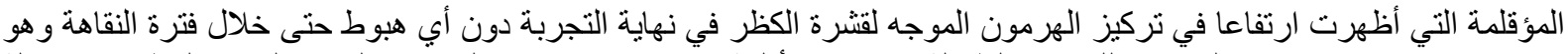

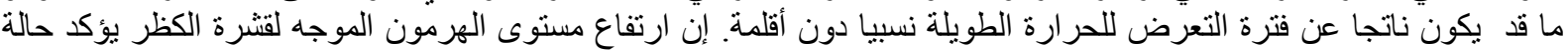

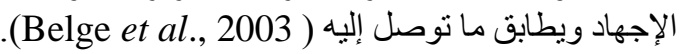

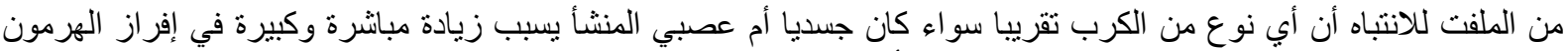

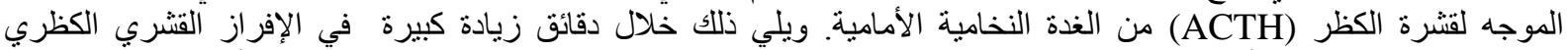

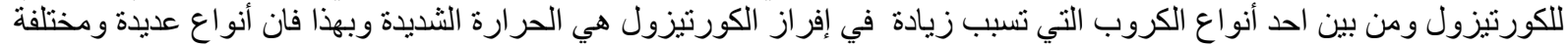

من المنبهات تتمكن من ان نولد زيادة كبيرة في سرعة إفراز الكورتيزول من قترة الكظر (Guyton and Hall, 2006).

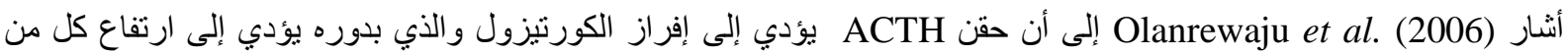
O2,PCo2,K+1 ,Na +1, Ca

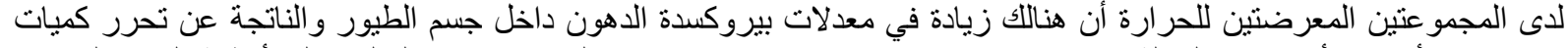

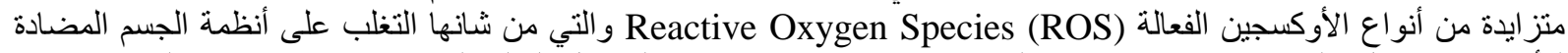

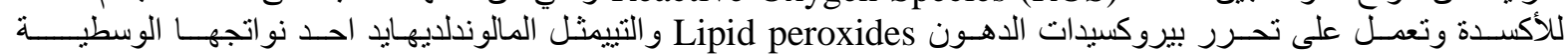

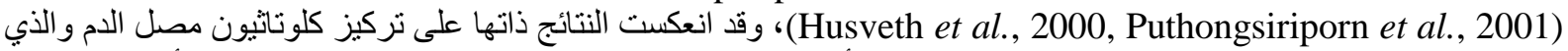

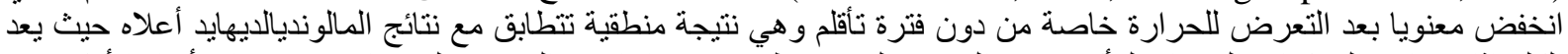

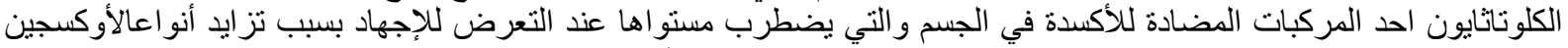

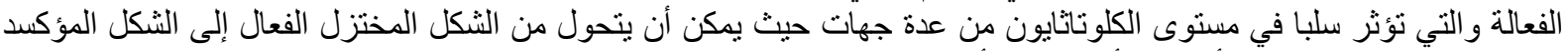

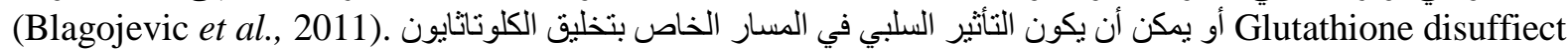




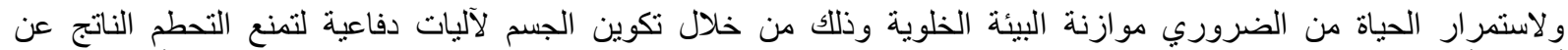

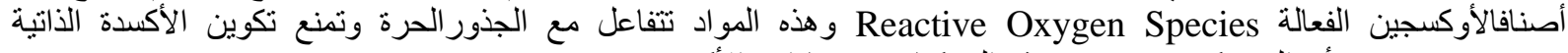

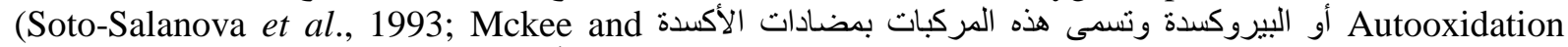
ولتقليل الاضطر ابات الخلوية و الحد من تأثير ات الإجهاد ينم من خلال هذه المواد وتشمل الموازنة الداخلية للخلية. أن مضادات الأكسدة الدفاعية تتم من خلال خمس مر احل مختلفة وتثمل:

منع ايض الجذور وطرح منتجات الجذور فضلا عن إصلاح الخلايا وتثبيط سلسلة التفاعلات المنتجة للجذور الثانوية وزيادة

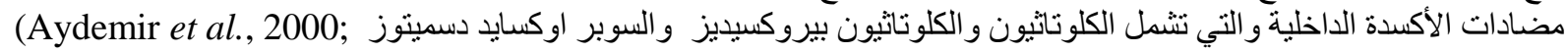
Sahin et al., 2002; Ruiz et al., 2001; Puthongsiripoorn et al., 2001)

أما بالنسبة للجدولرقم (Y) فقد لوحظ ان زيادة تركيز ايون البوتاسيوم في الدم يمكن أن تكون ناتجة عن عملية تخفيف الدم بسبب زيادة

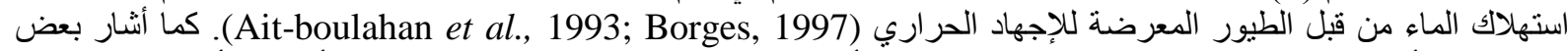
الباحثين إلى أن ايون البوتاسيوم الموجود داخل الخلايا يمكن أن يتحرر الى الدم بسبب تغير النير في نضوحية الأغشية الأمر الذب من شانه

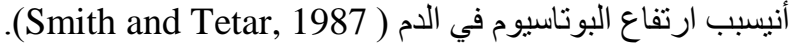

وقد أشار (Deyhim et al., 1990; Belay and Teeter, 1993; Ait-boulahsen et al., 1995; Borges, 1997) إلنأن

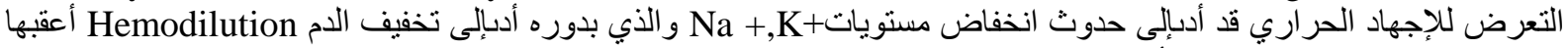

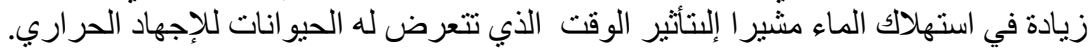

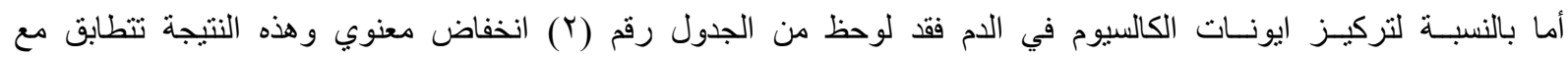
(Allahverdi et al., 2013; Taylor and Hertelendy, 1961; Parsons and Combs, 1981; Van de Velde et al., (1986 و الذي يمكن أن يكون نتيجة لحالة التهوية الفائقة Hyperventlation نتيجة الإجهاد الحراري للادجاج و التي ينتج عنها حالة

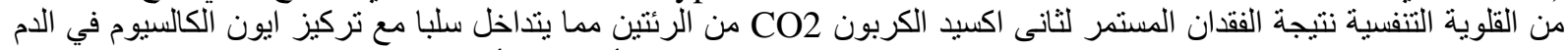

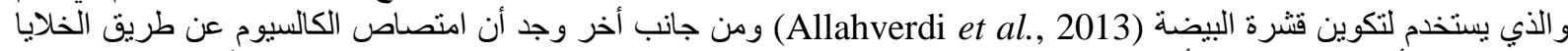
الظهارية في ألاثني عشر يمكن أن يقل إلى حد كبير نتيجة الإجهاد الحراري (Deaton et al., 1969) (محمود وآخرون ، 1996).

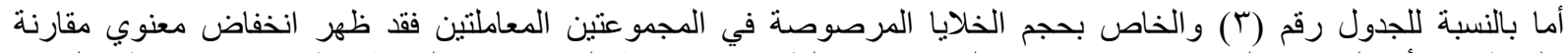

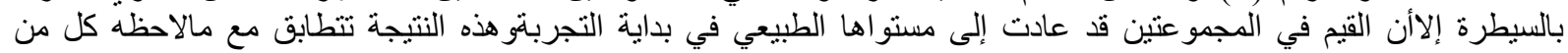
(Deaton et al., 1969; Kubena et al., 1972; Vo et al., 1978; Deyhim and Teeter, 1991; Yahav and

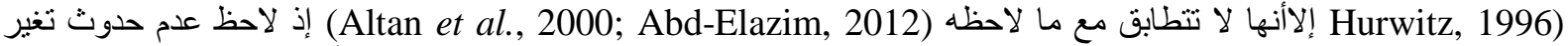

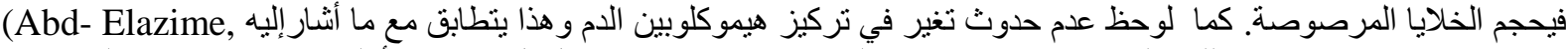

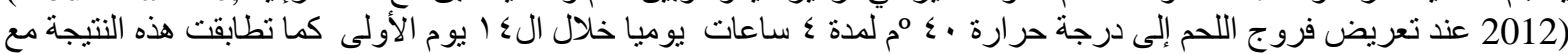
(Altan et al., 2000)

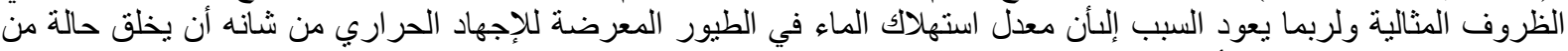

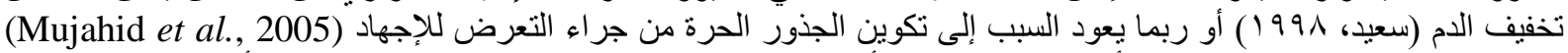

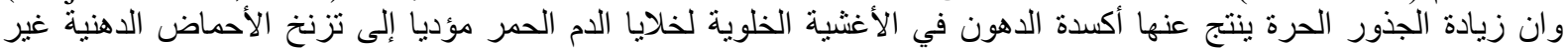

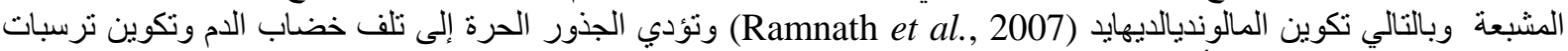

داخل خلايا الدم الحمر تدعى بأجسام هينز (Kumar et al., 1997).

أما سبب انخفاض معدل حجم الخلايا المرصوصة فربما يعود أيضاالىى حالة تخفيف الدم أو قد يعود السبب إلتالأذى التاكسدي للإجههاد

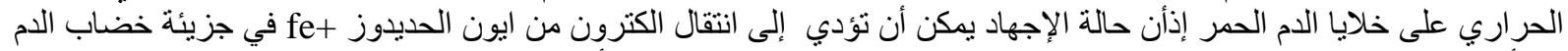

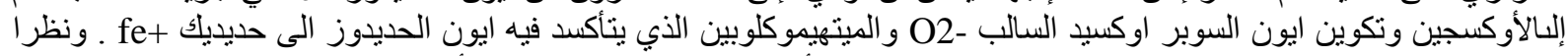

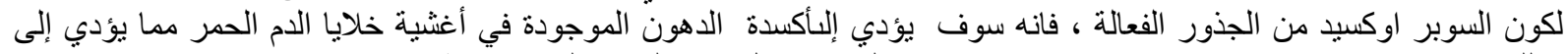

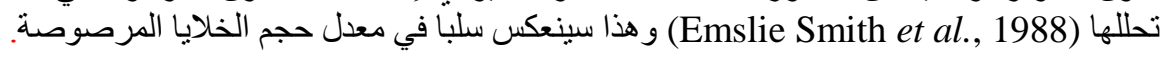

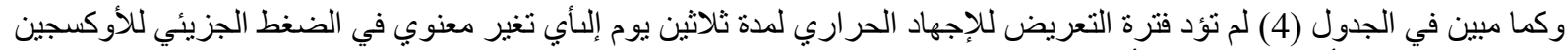

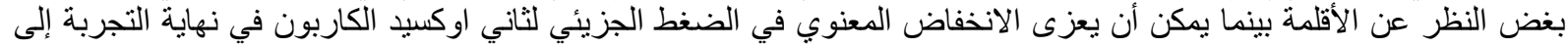

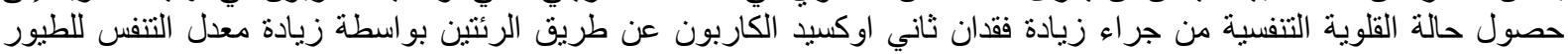

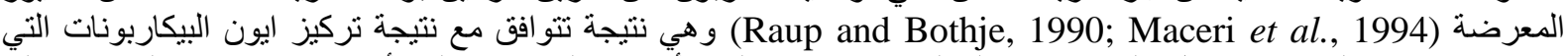
ارتفعت في نهاية التجربة مما يدل على حدوث حالة القاعدية وجدير بالذكر أن فترة النقاهة كان لها تأثير كبير في عودة الحيو التيوانات إلى الثي 


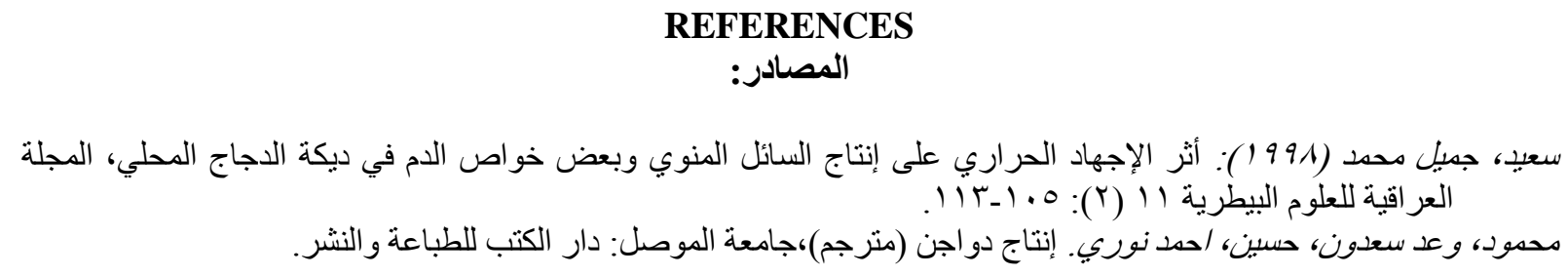

Abd-Elazim, A.M. (2012): Improve the heat tolerance of broilers through heat treatment during the first two weeks. Egypt. Poult. Sci. Vol. (32) (ш): (483-495).

Abd-Elsamee, M.O. (2005): Influence of different levels of lysine and vitamin (E) on broiler performance under normal or heat stress conditions. Egyptian J. Nutr. Feeds. 8:827-838.

Ait-boulashan, Glalich, J.D. and Edens, F.W. (1995): Potassium chloride improves the thermotolarance of chickens exposed to acut heat stress. Poult.Sci., 74; 75-78.

Allahverdi, A.; Feizi, A.; Takhtfooladi, H.A. and Nikpiran, H. (2013): Effects of Heat Stress on Acid- Base Imbalance, Plasma Calcium Concentration, Egg Production and Egg Quality in Commercial Layers. Global Veterinaria 10(2):203- 207.

Altan, O.; Altan, A.; Cabuk, M. and Bayraklar, H. (2000): Effect of heat stress on some blood parameters in broiler. Turk. J. Vet. Anim.Sci., 24: 145-148.

Aydemir, T.; Ozturk, R.; Bozkaya, L.A. and Tarhan, L. (2000): Effects of antioxidant vitamins A,C, E and trace elements $\mathrm{Cu}$, Se on CuZn SOD, GSH- Px, CAT and LPO levels in chicken erythrocytes. Cell Biochem. Funct.18, 109-115.

Blagojevic, DP.; Grubor-Lajsic, GN. and Spasic, MB. (2011): Cold defence responses: the role of oxidative stress. Front Biosci. 1;3: 416-27.

Belay, T. and Teeter, R.G. (1993): Broiler water balance and thermobalance duringthermoneutral and performance during heat stress. Applied Poult Res., 3 (1): 87-92.

Belge, F.; Cinar, A. and Selcuk, M. (2003): Effect of stress produced by adrenocorticotropin(ACTH) on lipid peroxidation and some antioxidants in vitamin $\mathrm{C}$ treated and non treated chickens. South African Society for Animal Science, 33(3), 201-205.

Beuge, J.A. and Aust, S.D. (1978): Estimation of Serum Malondialdehyde Level. Academic Press, London, pp: 34-36.

Bishop, M.L.; Fodly, E.P. and Schoeff, L. (2005): Clinicalchemistry. $5^{\text {th }}$ ed. ippincott Williams and Wilkins, A. Wolters Kluwer company., pp: 205-626.

Borges, S.A. (1997): Suplementacao de cloreto de potussioe bicoarbonato de sodio para Frangos de cortedurante o Verao. Dissertaca.De mestardo, UNESP, Jaboticubal, Brazil.

Borges, S.A.; Fisher da Silva, A.V.; Ariki, J.; Hooge, D.M. and Cummings, K.R. (2003): Dietary electrolyte balance for broiler chickens under moderately high ambient temperatures and humidity. Poult. Sci. 82: 301-308.

Bruits, C.A. and Ashood, E.R. (1999): Tietz-textbook of clinical chemisty. W.B. Saunder Company.

Deaton, J.W.; Reece, F.N. and Tarver, W.J. (1969): Hematocrit, hemoglobin and plasma protein levels of broilers reared under constant temperatures. Poult. sci., 48: 1993-1996.

Deyhim, F. and Teeter, R.G. (1991): Sodium and potassium chloride drinking water supplementation effects on acid -base balance and plasma corticosterone in broiler reared in thermoneutral and heat- distressed environments. Poult.Sci.70: 2551-2553.

Deyhim, F.; Belay, T. and Teeter, R.G. (1990): The effect of heat distress on blood gas, plasma and urine concentration of $\mathrm{Na}, \mathrm{K}, \mathrm{Cl}$ of broiler chicks. Poult. Sci. 69: 42(abstr.).

Dohms, J.E. and Metz, A. (1991): Stress- mechanisms of immunosuppression. Vet. Immunol. Immunopathol, 30: 89-109.

Emslie-Smith, D.; Paterson, C.R.; Seratvcherd, T. and Read, N. (1988): (Eds). Churchill, Livignston, Edinburgh. Guyton, A.C. and Hall, J.F. (2006).Text book of medical physiology. $11^{\text {th }}$ ed., Elsevier science, Philadelphia.

Husveth, F.; Manilla, H.A.; Gaal, T.; Vajdovich, P.; Balogh, N.; Wagner, L.; Loth, I. and Nemeth, K. (2000): Effects of saturated and unsaturated fats with vitamin $\mathrm{E}$ supplementation on the antioxidant status of broiler chicken tissues. Acta. Vet. Hung. 48, K. 69-79.

Kubena, L.F.; May, J.D.; Reece, F.N. and Deaton, J.W. (1972): Hematocrit and hemoglobin levels of broilers as influenced by environmental temperature and dietary iron level. Poult. Sci. 51: 759-763.

Kumar, V.; Cotran, R. and Stanley, L.R. (1997): Baisc pathology. $6^{\text {th }}$ ed., WB. Saudrescompany. Philadelphia.

Maceri, M.R.; Furlan, L. and Gonzales, E. (1994): Fisiologiaaviariaaplicada a frangos de corte. FUNEP/UNESP, Jaboticabal, Brazil. 
Matta, J.; Milad, M.; Manger, R. and Tosteson, T. (1999): Heavy metals, lipid peroxidation, and ciguatera toxicity in the liver of the Caribbean barracuda (Sphyraena barracuda). Biol. Trace Elem. Res.; 70(1): 69- 79.

McKee, J.S. and Harrison, P.C. (1995): Effects of supplemental ascorbic acid on the performance of broiler chickens exposed to multiple concurrent stressor. Poult.-Sci.74, 1772-1785.

Mujahid, A.; Yoskiki, Y.; Akiba, Y. and Toyomizu, M. (2005): Superoxide Radical production in chicken skeletal muscle induced by acute heat stress. Poult. Sci., 84: 307-314.

$N R C$, (1994): Nutrient Requirements of Poultry. $9^{\text {th }}$ revised Ed. National Reseach Council, Washington, USA.

Olanrewajuetal, H.A.; Wongpichet, S.; Thaxton, J.P.; Dozier w, W.A. and Branton, S.L. (2006): Stress and acid base balance in chickens. Poult. Sci., 85: 1266-1274.

Parson, A.H. and Combs, G.F. (1981): Blood ionized calcium in the chicken. Poult. Sci.,53: 1520-1524.

Puvaldopirod, S. and Thaxon, J.P. (2000): Model of physiological stress in chickens. 2. Dosimetry of adrenocorticotropin. Poult. Sci. 80, 976-82.

Puthongsiriporn, U.; Scheideler, S.E.; Sell, J.L. and Beck, M.M. (2001): Effects of vitamin E and C supplementation on performance, in vitro lymphocyte proliferation, and antioxidant status of laying hens during heat stress. Poult. Sci. 80, 1190-1200.

Ramnath, V.; Rekha, P.S. and Sujatha, K.S. (2007): Amelioration of heat stress induced disturbances of antioxidant defense system in chicken by BarhmaRasayana. eCAM., 10:1-8.

Raup, T.J. and Bothje, W.G. (1990): Effect of carbonated water on arterial PH, Pco2 and plasma lactate in heatstressed broilers. Br. Poult. Sci. 31: 377-348.

Rozenboim, I.; Mobarky, N.; Heiblum, R.; Chaiseha, Y.; Kang, S.W.; Biran, I.; Rosenstrauch, A.; Sklan, D. and El-Halawani, M.E. (2004): The role of prolactin in reproductive failure associated with heat stress in the domestic turkey. Biol. Reprod. 71: 1208-1213.

Ruiz, J.A.; Guerrero, L.; Arnau, J.; Guardia, M.D. and Esteve-Garcia, E. (2001): Descriptive sensory analysis of meat from broilers fed diets containing vitamin $\mathrm{E}$ or beta-carotene as antioxidants anddifferential fats. Poult. Sci. 80, 976-82.

Sahin, K.; Sahin, N. and Yaraliogl, U. (2002): Effect of vitamin and E on lipid peroxidation, blood serum metabolites, and mineral concentrations of laying hens reared at high ambient temperature. Biol. Trace Elem. Res. 85, 35-45.

Salvador, D.; Ariki, J. and Borges, S.A. (1999): Suplementagao de bicarbonate de sddionaragao a naagua de bebida de frangos de cortesubmetidosaoestressesalorico. ARS Veterinaria. 15: 144-148. (Abstr.).

Siegel, H.S. (1985): Immunological response as indicators of stress. World's Poult. Sci. J.41: 36-43.

Smith, M.O. and Teeter, R.G. (1987): Potassium balance of 5to 8-week- old broiler exposed to constant heat or cycling high temperature stress and the effects of supplemental potassium chloride on body weight gain and feed efficiency. Poult. Sci. 66: 487-492.

Sotosalanova, M.F.; Sell, J.L.; Mallarino, E.G.; Piquer, F.J.; Barker, D.L.; Palo, P.E. and Ewan, R.C. (1993): Research note: vitamin E status of turkey poults as influenced by different dietary vitamin E sources, a bile salt, and an antioxidant. Poult. Sci. 72, 1184-1188.

Steel, R.G. and Torrie, J.H. (1980): Principle and Procedures of Statistics (2 ${ }^{\text {nd }}$ ed.). McDonald book Co., Inc., New York.

Taylor, T.G. and Hertelendy, F. (1961): Changes in the Blood Calcium Associated with Egg Shell Calcification in the Domestic Fowl:2. Changes in the Diffusible Calcium Poult. Sci., 40:115-123; doi: 10.3382/ps. 0400115.

Trout, J.M. and Mashaly, M.M. (1994): The effects of adrenocorticotropic hormone and heat stress on the distribution of lymphocyte populations in immature male chickens. Poult. Sci.73, 1694-1698.

Van de Velde, J.P.; Van Grinkel, F.C. and Vermeiden, J.P.W. (1986): Patterns and relationships of plasma calcium, protein and phosphorus during the egg laying cycle of the fowl and the effect of dietary calcium. Br. Poult. Sci., 27: 421-433.

Vo, K.V.; Bone, M.A. and Johonson, W.E. (1978): Effect of three life time ambient temperatures on growth, feed and water consumption and various blood components in male and female leghorn chickens. Poult. Sci. 57: 798-803.

Wierusz-Wysockaa, B.; Wysocki, H.; Byks, H.; Zozuliriska, D.; Wykr\#owiczb, A. and Kahnierczak, M. (1995): Metabolic control quality and free radical activity in diabetic patients. Diabetes Research and Clinical Practice 27 (1995)193-197.

Yahav, S. and Hurwitz, S. (1996): Induction of thermotolerance in male broiler chickens by temperature conditioning at any early age. Poult. Sci. 75: 402-406.

Brown, R.L.; Bhatnagar, D.; Cleveland, T.E. and Cary, J.W. (1998): Recent advances in preharvest prevention of mycotoxin contamination, p. 351-379. In K.K. Sinha and D. Bhatnagar, (ed.).

Brown, R.L.; Bhatnagar, D.; Cleveland, T.E. and Cary, J.W. (1998). Recent advances in preharvest prevention of mycotoxin contamination, p. 351-379. In K. K. Sinha and D. Bhatnagar, (ed.). 